\title{
Los desafíos en la formación de los futuros médicos
}

\author{
Challenges in the training of future doctors
}

Ricardo Teodoro Ricci ${ }^{\ddagger}$

\begin{abstract}
Resumen
En este artículo, el autor reflexiona sobre sobre el presente y el futuro de la educación médica en el contexto de las tecnologías de la información y de la convivencia de nuestro trabajo con los dispositivos de inteligencia artificial, de los nuevos contenidos de genética y neurociencias, del trabajo en equipo y de la necesaria resignifificación de los términos "cura" y "cuidado". Se pregunta además si estamos en condiciones de encarar dicho desafío y de estar a la altura de las necesidades educativas de las próximas (y la actual) generación/es de médicos.
\end{abstract}

\section{Abstract}

In this article, the author reflects on the present and the future of medical education in the context of information technologies and the coexistence of our work with artificial intelligence devices, the new contents of genetics and neurosciences, about team work and the necessary resignification of the terms "cure" and "care". He also wonders if we are able to face this challenge and to deal with the educational needs of the next (and the current) generation/s of doctors.

Palabras clave: educación médica, inteligencia artificial, trabajo en equipo, cura, cuidado. Key words: medical education, artificial intelligence, teamwork, healing, care

Ricci RT. Los desafíos en la formación de los futuros médicos. Evid Act Pract Ambul. 2018. 21(3) 70-72.

\section{Las tecnologías de la comunicación}

Lo primero es hacer una advertencia rotundamente evidente y es que el siglo XXI hace ya tiempo que comenzó y ya se encuentra avanzado. Eso implica que ya deberíamos tomar con naturalidad la instalación definitiva de las tecnologías de la comunicación, los cambios culturales impuestos por los millenials y el notable vértigo de todos los procesos sociales en los que nos hallamos implicados, entre otros el que compromete a la misma Educación Médica. Se trata concretamente de una época de cambio constante que conlleva modos concretos y fugaces de adaptación, por lo tanto, el desafío constante es el de la anticipación. Ocurre que a menudo los cambios hechos en la educación médica han sido tibios, descomprometidos y autoboicoteados con retroalimentaciones negativas. Considero que cierto "gatopardismo" tiende a imponerse en nuestras instituciones educativas: "Cambiar mucho para que en realidad nada cambie".

Resulta evidente que nunca hubo tanta propuesta y estrategia de cambio, y/o tantas experiencias educativas en el terreno. Sin embargo, frecuentemente advertimos que lidiamos con los mismos problemas que ya considerábamos solucionados. Nos invade la sensación de que no podemos terminar de cambiar porque ya otro cambio nos seduce y nos distrae de nuestros genuinos objetivos. Ese es un enorme signo del siglo y a la vez, un enorme llamado a la prudencia y a la serenidad.

\section{Los contenidos de la genética y de la neurociencias}

Con respecto a los contenidos, el siglo ya nos está desafiando a adoptar posiciones y a incluir en los planes de estudios temas nucleares de Genética y de Neurociencias. Las ciencias relacionadas con la medicina nos están proponiendo insistentemente estas dos disciplinas de desarrollo vertiginoso.

El Proyecto Genoma Humano ha dado enormes pasos, y con la manipulación y la edición genética, estamos de lleno en un presente-futuro del que por ahora la academia parece ajena. No nos estamos adecuando con la debida premura.

Las neurociencias por otro lado, con el Proyecto Cerebro Humano, están a las puertas de develar el otro gran misterio del siglo: la conciencia humana. Se está a unos pocos pasos de mapear todo el cerebro humano y de identificar el diseño del Conectoma, es decir el aparato de conexión de las áreas ce-rebrales de la sustancia blanca. Esto, que dicho de esta manera casi resulta anodino, nos desafía frontalmente ya que se está hablando de temas acuciantes como la modificación de memorias, el acrecentamiento de procesos perceptivos, y el incremento de las funciones ejecutivas cerebrales por medio de drogas y de estimulación transcraneana. Una serie de intervenciones que, al igual que la genética, hagan del ser humano que conocemos un mero antecedente de un hombre pos-humano que la medicina deberá estar en condiciones de asistir. En este contexto los desafíos antropológicos y bioéticos pueden ser calificados de extremos.

\section{De la cura al cuidado}

En la práctica clínica se está definiendo de manera clara un trayecto que va desde la cura hacia el cuidado. El origen latino de ambas palabras es idéntico y ambas se refieren concretamente al cuidado. Sin embargo, en nuestro léxico médico son dos palabras que connotan realidades muy diferentes. Los que hemos sido formados durante la segunda mitad del siglo XX hemos desarrollado el optimismo de la cura ya que los recursos diagnósticos y terapéuticos que de pronto la medicina tuvo a su disposición, nos permitieron envalentonarnos con la cura de las enfermedades. Esa realidad rindió sus frutos y cambió de tal modo el escenario, que hoy nuestros alumnos necesariamente deberán hacer hincapié en el cuidado.

Con esto quiero decir que: la enfermedad y el padecimiento del siglo presente se orientan a la patología crónica y degenerativa, a la ancianidad, la minusvalía, a los deterioros cognitivos, etc. En ese contexto es imprescindible proveer a nuestros futuros médicos con competencias que a nosotros nos resultaban secundarias o 'blandas'. Concretamente, se trata de las competencias comunicacionales y narrativas, de acompañamiento empático diseñado a largo aliento, de atención a los detalles, de disponibilidad y escucha, y de apertura de canales de comunicación fluidos en el tándem familia-equipo de salud, que debe funcionar como una unidad. Si los futuros profesionales de la salud desean estar a la altura de lo que se requiere de ellos, deberán ser formados con habilidades y competencias de liderazgo y compromiso, ya que no se entiende otro modo de enfrentar las realidades mencionadas como no sea con un coordinado trabajo en equipo.

El trabajo en equipo

‡ Departamento de Antropología Médica. Facultad de Medicina de la Universidad Nacional de Tucumán. teodoro.ricci@gmail.com 
Nuestros alumnos deberán estar preparados para respetar como compañeros de equipo a kinesiólogos, enfermeros, nutricionistas, acompañantes terapéuticos, cuidadores, diseñadores de espacios domiciliarios de atención médica, y a muchos otros modos de prácticas terapéuticas que aún no se nos ocurren. Ese equipo necesita líderes comprometidos y claros en sus objetivos. La tarea de proveer de atención a este tipo de enfermos genera un altísimo costo sanitario por lo que nuestros profesionales deberán estar preparados para la máxima eficacia con máxima austeridad.

Nos encontramos ante la perentoria necesidad de implementar comunidades de aprendizaje y práctica en las que se valore la interdisciplina, en la que los contextos y la ecología de lo humano marquen el camino de lo correcto. Comunidades educativas de alto compromiso en las que se reconozca el valor de lo explícito y también de lo implícito. Los docentes deben ser formados para percibir la importancia de ambas formas de transmitir conocimientos, competencias y valores.

\section{La convivencia con los dispositivos de inteligencia artifical}

Otro desafío del porvenir que ya se encuentra entre nosotros es la tendencia a la sustitución del médico como agente principal de la salud de las personas y las sociedades. El médico va a ser reemplazado por las máquinas en la mayoría de sus tareas diagnósticas y terapéuticas, y mucho más en aquellas de promoción de la salud y la prevención. Además, muchos asuntos pasarán a ser de dominio del público y se dirimirán a través de las redes sociales, aún hoy incipientes. Agentes no diplomados dedicados a diversas formas de terapias alternativas o no convencionales ocuparán poco a poco la mayoría del espacio de la patología leve, las dependientes de inconsistencias higiénico-dietéticas y las vinculadas con la actividad física.

Lo que hoy conocemos con diagnóstico clínico será reemplazado por máquinas de Inteligencia Artificial que ejecutarán algoritmos de mediana y alta complejidad. Poco a poco seremos reemplazados para aquellas tareas sencillas y rutinarias que los médicos reclamamos como propias. El desafío consiste en superar con dignidad y altura epistemológica ese embate sustancial ya que considero que el profesional de la salud nunca podrá ser reemplazado como el agente empático que conoce de dolores y aflicciones, ni en términos de brindar compasión y comunicarse con un semejante. Me animo a asegurar que, por ahora, tampoco podrá ser sustituido en actividades que comprometan la originalidad, la singularidad y la particularidad del caso único§.

Para responder a estos desafíos se hace imprescindible la potenciación cualitativa de los profesionales de la salud. La academia deberá aprender a privilegiar las diferencias y las originalidades creativas y desterrar las homogeneidades de pensamiento único a las que está acostumbrada. Las competencias más valiosas serán la capacidad de escucha, y la de generar escenarios de participación responsablemente comprometida entre pacientes y equipos de salud. Saber cómo diseñar escenarios de reconfiguración y resignificación del sufrimiento, de la enfermedad y de la muerte serán competencias destacadas y apreciadas.
¿Perdurarán los médicos y el resto de las profesiones vinculadas a la atención de la salud de las personas en medio de tanta tecnología? Claro que sí. El personal humano programará al detalle las máquinas y las manejará con pericia en las instancias asistenciales, pero permanecerán lejos de los pacientes.

Con el 'enfermo' deberá estar un individuo comprometido, prudente, justo, atento y de mentalidad abierta que, implicándose, acuerde con los pacientes y sus entornos la creación de cursos alternativos de acción que los ayude a salir adelante.

\section{¿Quién se encargará de semejante tarea?}

Finalmente siento el deber de hacer una fugaz semblanza del desafío que considero más antiguo, complejo y a la vez más temido. Para tratarlo recurro al sistema de un viejo profesor de filosofía: Él la denominaba la técnica de las cinco "W" y una "H". Consiste sencillamente en construir la descripción de un asunto determinado respondiendo a las siguientes preguntas: Qué, Cuándo, Dónde, Por y Para qué, Quién y Cómo (What, When, Where, Why, What For and How).

Para responder a algunas de estas preguntas en la educación médica la bibliografía es sobreabundante y también lo son las experiencias compartidas en eventos científicos y foros como el Congreso Anual de Educación Médica. No cabría esperar algo distinto. Sin embargo, continúo percibiendo como un gran desafío para la educación médica a la pregunta por el Quién (Who).

El fundamental reto al que nos enfrentamos es el de tener buenos docentes. No podemos pretender tener egresados excelentes si vemos a los docentes que los forman como meros empleados públicos. La formación de los profesores mediante carreras de formación docente y maestrías no es suficiente. Sabemos que el rol docente es complejo y variado. Somos conscientes de que posicionarse frente al alumno es sólo uno de los aspectos a tener en cuenta. Es conveniente recordar los otros: mantener una actualización permanente, enseñar desde la mejor evidencia, facilitar el aprendizaje, programar el desempeño, establecer etapas y metas, y algo extremadamente importante: dar testimonio permanente del rol de médico y de docente.

\section{Reflexiones finales}

Para ir concluyendo deseo proponer al respecto, dos líneas de consideración.

Primero: Para ser docente en una facultad de medicina hay que tener la vocación bifronte de médico y de maestro. Para que esa vocación se manifieste en plenitud es imprescindible contar con compromiso, con disponibilidad y con tiempo exclusivo para la tarea. No es suficiente con ser docente 'de paso', 'mientras hago otra cosa' o 'en los ratos libres'.

Segundo: Es menester contar con un sistema de evaluación docente permanente y exigente, algo que se parezca a una evaluación de desempeño. Como responsable de una cátedra me

$\S$ Por otro lado, los avances tecnológicos tienen para nosotros otro desafío letal. Si puedo graduarme en Stanford, en Princeton o en Utrech, ¿por qué habría de hacerlo en Tucumán? La sociedad del conocimiento está engendrando grandes monstruos educativos, como lo estamos experimentando con los posgrados. Las posibilidades on-line son infinitas y conspiran contra aquellos que no perciban esta realidad y no sean capaces de diferenciarse cualitativamente de manera creativa, personalizada y adecuada a lo regional y particular. Estamos en plena transición hacia oligopolios, por no decir monopolios educativos. Por lo tanto, en este contexto global, consideramos perentoria la cooperación y el trabajo conjunto de nuestras unidades académicas para que puedan crear valor priorizando lo original del trabajo en cada región. 
percibo indulgente por no evaluar seria, rigurosa y permanente a los docentes que la integran. No pondero convenientemente la opinión de los alumnos, no juzgo con rigor la falta de participación en programas docentes, en cursos optativos, en programas de investigación, en voluntariados o en aspectos de gestión. No tengo un plan de evaluación, del mismo modo que la institución no posee una sistemática para evaluarme a mí como responsable de dicha cátedra. Haciendo un mea culpa advierto que esa referida indulgencia corre el enorme riesgo de transformarse en negligencia.
Este es un tema que me preocupa en demasía. Soy testigo permanente de la levedad con que la mayoría de los colegas docentes encaran su labor. Sé que, tocando este evitado tema, estoy abriendo una caja como la de Pandora, sin embargo, también sé que en su fondo está la esperanza.

Mientras tanto siento que estamos siendo testigos y protagonistas de muchas, quizás demasiadas miserias.

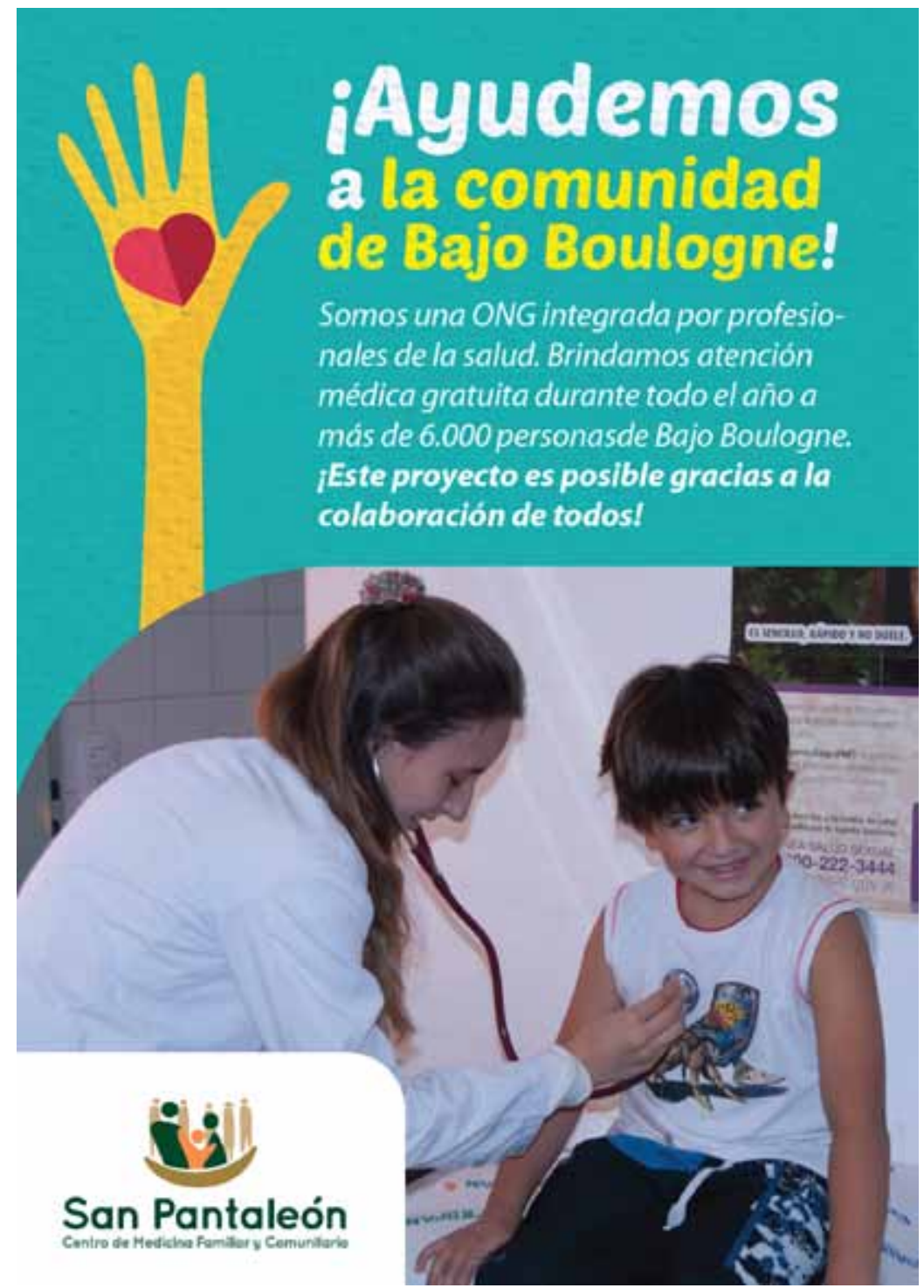

